\title{
Concordance between the WCRF recommendations and reduced global cardiovascular risk in a cohort of survived breast cancer patients
}

\author{
Simone Magro ${ }^{1}$, Francesco Sofi ${ }^{2}$, Gabriele Mascherini ${ }^{1}$, Giorgio Galanti ${ }^{1}$ and Laura Stefani ${ }^{*}$ \\ ${ }^{1}$ Department of Experimental and Clinical Medicine, Unit of Sports Medicine, University of Florence, Italy \\ ${ }^{2}$ Department of Experimental and Clinical Medicine, Unit of Clinical Nutrition, University of Florence, Italy
}

\begin{abstract}
Background: body weight control by proper diet management following the World Cancer Research Fund recommendations (WCRF) and physical activity play a role in reducing cancer risk and improving quality of life. The study evaluates the concordance between the WCRF recommendations, updated to 2017, and the improvement of anthropometric and physical parameters in a cohort of patients with previous stable breast cancer, without signs of co-morbidity and in complete remission of neoplastic disease, in Italy.
\end{abstract}

Methods: this is an open, randomized, controlled, parallel clinical trial. Twenty-one patients with previous breast cancer were recruited at the Department of Sports Medicine and Exercise of the University Hospital, Florence. The patients were divided into two groups (Group 1 and Group 2). They were submitted to two evaluations in a week: in the first a complete evaluation was performed in terms of body composition, physical performance, myocardial conditions and adherence to the Mediterranean diet. In the end, a weekly food diary was delivered to understand patient habits. In the second visit, after the completion of the food diary, an in-depth analysis on nutrition was carried out. Lifestyle and nutrition were corrected for G1 using WCRF recommendations; instead, general and standardized food councils have been delivered to G2. The follow-up was two months for patients of both groups, in which all anthropometric, physical and dietary measures were repeated.

Results: Significant improvements were observed in G1 for the following anthropometric parameters: BMI -0.4 (from 28.6 to 28.2; 95\% CI), weight -1.0 kg (from 72.7 to $71.7 ; 95 \% \mathrm{CI}$ ), waist circumference $-1.7 \mathrm{~cm}$ (from 93.5 to $91.8 ; 95 \% \mathrm{CI}$ ) and hip circumference $-1.3 \mathrm{~cm}$ (from 104.9 to 103.6 ; $99 \% \mathrm{CI}$ ). Adherence to the Mediterranean diet, determined with the MEDI-LITE score, was increased by 1 point (from 14 to 15; 99\% CI). Finally, significant improvements were identified for the amount of weekly physical activity, equal to 35 minutes (from 173 to 208; $99 \% \mathrm{CI}$ ) and for strength of the lower body, determined with the Chair Test, equal to two repetitions (from 17 to $19 ; 95 \% \mathrm{CI}$ ).

Conclusions: The study suggests that the following WCRF recommendations could significantly improve most of the anthropometric and physical parameters among female breast cancer survivors. It can be widely proposed in populations.

\section{Introduction}

Cancer is the second leading cause of death, and accounts for about $30 \%$ of causes of death in reference to the global population [1]. The age of cancer onset is prevalent in the range between 30 and 60 years, when co-morbidities more easily associated to an incorrect lifestyle. It is known that in chronic degenerative diseases, lifestyle adaptation and adherence to the Mediterranean diet, especially in those with previous neoplastic pathology, lead to a reduction of cardiovascular risk, as well as a reduction in the appearance of morbidity [2]. It is therefore reasonable to promote correct life style to prevent a significant percentage of cancer diseases. Especially over the last 30 years the weight control by the right dose of physical activity and correct diet support the success of primary prevention, for cancer. Moreover, the post-chemotherapeutic phase is in many cases associated with an increase in body weight [3]. However, the results of several studies have shown that some of the well-known scoring indexes have minimal or no association with the risk and/or overall mortality of cancer.

However, in 2007, the World Cancer Research Fund and the American Institute for Cancer Research (WCRF/AICR) issued eight general and two special recommendations concerning diet, physical activity and weight management for cancer prevention, based on the most complete collection of evidence available today, including more than 7,000 scientific studies published on cancer prevention in the last 50 years. To date, more and more evidence of association between these 10 recommendations and the overall reduction of risk and mortality from cancer is accumulating, thanks to important publications, such as EPIC. The study aims to verify in a group of breast cancer patients, following an exercise prescription program, the impact of the WCRF recommendations on the principal anthropometrics parameters related to the cardiovascular risk factors.

${ }^{\star}$ Correspondence to: Laura Stefani, Department of Experimental and Clinical Medicine, Unit of Sports Medicine, Via delle Oblate, 50141, Florence, Italy, E-mail: laura.stefani@unifi.it

Key words: breast cancer, diet, nutrition, weight control, physical activity, quality of life

Received: April 08, 2019; Accepted: April 23, 2019; Published: April 27, 2019 


\section{Materials and methods}

\section{Study design}

The study was randomized, controlled, open-label, in parallel clusters. A group of 21 patients with previous breast cancer were recruited and followed at the Sports Medicine and Exercise Center of the University of Florence-Italy. All they were in stable conditions and complete remission of cancer disease and all participating in the moderate exercise program (aerobic and against resistance) following the guidelines of the American College of Sports Medicine.

At the beginning of the study they were randomized to two intervention groups:

- Group 1 - following the WCRF/AICR recommendations.

- Group 2 - following general dietary advice, without including the WCRF/AICR recommendations.

The 21 patients involved were already present in the archives of the Department of Sports Medicine and Exercise and most of them were periodically followed every six-monthly basis, for the check-up. They were contacted by telephone and proposed to participate in the intervention study. After signing informed consent, 10 participants were assigned to Group 1 and 11 to Group 2.

Group 1 recommendations were administered by the WCRF/AICR, tailored to the patient's specific habits and to any co-morbidities present, and the preventive importance of the 10 behaviours in the reappearance of relapses was explained.

Group 2 was invited to follow the general diet advice, taking as a reference the Mediterranean way of life:

- Divide the food intake into the day into five meals, including three main meals and two snacks.

- Consume carbohydrates at lunch, combining them with at least a portion of vegetables preferably.

- Take preferably proteins at dinner, varying the type, associating them with at least a portion of vegetables.

- For snacks, preferably consume a fruit or a yogurt.

For both the groups, two check-ups were planned, one at the beginning of the study and one after two months of intervention. The parameters examined were:

- Anthropometric parameters: BMI, waist circumference, tricipital, bicipital, subscapular, suprailiac skin folds.

- Body composition was evaluated by bioimpedance analysis with measure of lean mass (FFM), fat mass (FM), total water.

- Upper and lower strength parameters resulted from physical tests: Hand Grip Test, Sit \& Reach Test (SRT), Chair Stand Test (CST), 6 'Minute Walking Test (6' MWT).

- Adherence to the Mediterranean Diet, using the MEDI-LITE Score questionnaire [4].

At the end of the first check-up a weekly food diary was also given. They were asked to declare the food consumed during the day, divided by time, quantity, place of consumption and emotions associated with them; it was requested to specify, moreover, the daily quantity of water taken, any physical activity performed, specifying the type and duration, and the time of sedentary activity. After this time, food diary was returned, habits regarding diet and lifestyle were analysed.

In order to correctly establish the tailored exercise to prescribe, the cardiovascular resistance and response skills were assessed by the 6 Minute Walking Test. In addition, the femoral and lumbar extensibility with the Sit \& Reach Test, the power in the lower limbs with the Chair Stand Test and strength of the intrinsic and extrinsic muscles of the hand with the Hand Grip Test.

At the end of the visit the adherence to the Mediterranean Diet of patients was evaluated using the MEDI-LITE score [4], which gives a score from 0 to 18 according to the frequency of consumption of nine categories of food: fruit, vegetables, cereals, legumes, fish, olive oil, meat and meat products, dairy products and alcohol. For the food groups typical of the Mediterranean diet, i.e. fruits, vegetables, cereals, legumes, fish and olive oil, a score of 2 was assigned for the maximum consumption frequency, 1 for the average intake category and 0 for the lowest consumption dose; on the contrary, for meat and dairy products, i.e. for non-Mediterranean food groups, an inverse score was awarded, based on a maximum value of 2 for the lowest consumption frequency, 1 for intermediate consumption and 0 for the highest recruitment category. For alcohol, a score of 2 was assigned for the intermediate consumption rate, 1 for the lowest intake category and 0 for the highest consumption dose. The more the score approached the maximum of 18 , the more the patient was adherent to the Mediterranean Diet. It was therefore possible to know the basic eating habits of the patient, comparing them with the Mediterranean diet and lifestyle, to date the one with the greatest scientific evidence in the prevention of chronic degenerative diseases [5].

\section{Anthropometric parameters}

The anthropometric parameters were calculated at the start and during the final phase of the study. Body composition parameters included weight, height and body mass index (BMI). Also, the hydration status was evaluated by bioimpedance analysis using the model BIA101 (Akern RJL Systems, Florence, Italy). Total body water (TBW), intracellular water (ICW) and extracellular water (ECW) were also measured.

For estimating fatty mass, the skinfolds were examined. They were measured using a Holtain caliper (Holtain Tanner/Whitehouse Skinfold Caliper, Holtain Ltd.) specifically at chest, mid axilla, abdomen, suprailium, front thigh, subscapular and tricipital levels. Body composition was calculated using the skinfold method. Also, fat mass (FM) and free fat mass (FFM) percentages were obtained by the Siri formula [6].

\section{Muscle strength and flexibility}

Muscle strength of the upper body was established following an indirect estimate by Hand Grip Test [7]. The Hand Grip Test consists of a muscle strength test using a hand gripper.

The Chair Test consisting in the maximum numbers of sitting in 30 seconds was used to evaluate the lower strength. The flexibility was evaluated by Sit $\&$ Reach.

\section{Statistical analysis}

The results were expressed as mean \pm standard deviation (SD) and calculated both at baseline (T0) and after two months (T1). The postpre intervention variations were expressed as the mean delta $\Delta \mathrm{T} 1-\mathrm{T} 0$ \pm DS. The differences between the two intervention groups were analysed with the Mann-Whitney test for independent samples, while 
the significance of the intra-individual variations was determined with the Wilcoxon test. The changes were considered statistically significant for $\mathrm{p}<0.05$.

\section{Results}

\section{Characteristics of the population}

Twenty-one women, average age of $56.7 \pm 8.7$ years, were examined. The characteristics of the total population and of the two intervention groups (Group 1 and Group 2) are shown in Table 1.

16 women (33\%) presenting an overweight condition and 5 (24\%) of obesity. The waist circumference was $88.6 \pm 13.2 \mathrm{~cm}$, indicating a state of visceral obesity and moderate risk of metabolic syndrome.

With reference to nutrition and lifestyle, the population had a good adherence to the Mediterranean diet, as demonstrated by the MEDILITE Mediterranean Diet questionnaire. In fact, based on a score ranging from 0 to 18 , the population has totalized $13 \pm 2$. Women have also declared to perform an average amount of physical activity weekly up to $186 \pm 121 \mathrm{~min}: 10$ of them (48\%) did not reach the 150 minutes recommended by the American College of Sports Medicine.
Group 1, consisting of the subjects who had received the advice of the WCRF/AICR, consisted of 10 women with an average age of $57.3 \pm 7.9$ years, while Group 2, made up of subjects who had received generic food indications, was composed of 11 women of average age equal to $56 \pm 9.6$ years. Although the Group 1 participants had weight, BMI, fat mass and waist circumference values greater than Group 2, no statistically significant differences emerged between the two groups for any of the parameters analysed (Table 1).

\section{Variation of the parameters}

After two months of interventions, the groups showed however different variations in terms of change of the main anthropometric parameters. Figure 1 shows the weight trend: for Group 1, the decrease was $0.8 \mathrm{~kg}$, with $\mathrm{p}=0.06$ and standard deviation (DS) $=1.1 \mathrm{~kg}$, while for Group 2 the variation was null, with $\mathrm{p}=0.97$ and $\mathrm{DS}=1.7 \mathrm{~kg}$. There were no statistically significant differences between the two groups ( $\mathrm{p}$ $=0.20$ ). Other important modifications have been observed in the two groups after the indications. They are shown in Table 2.

The modification of the BMI, reported in Table 2, confirmed the data related to the body weight change: in Group 1 the decrease was equivalent to $0.3 \mathrm{~kg} / \mathrm{m}^{2}$, with the same percentage change seen

Table 1: Characteristics of the two groups investigated at the onset of the study

\begin{tabular}{|c|c|c|c|c|}
\hline & $\begin{array}{c}\text { Total } \\
(n=21)\end{array}$ & $\begin{array}{c}\text { Group } 1 \\
(n=10)\end{array}$ & $\begin{array}{c}\text { Group } 2 \\
(n=11)\end{array}$ & p-value \\
\hline Age & $56,7 \pm 8,7$ & $57,3 \pm 7,9$ & $56,0 \pm 9,6$ & 0,60 \\
\hline Weight, kg & $68,6 \pm 14,8$ & $71,1 \pm 15,4$ & $66,2 \pm 14,7$ & 0,25 \\
\hline BMI, $\mathrm{kg} / \mathrm{m}^{2}$ & $26,8 \pm 6,0$ & $28,0 \pm 5,9$ & $25,8 \pm 6,1$ & 0,18 \\
\hline FM, \% & $32,8 \pm 11,0$ & $35,2 \pm 11,0$ & $30,6 \pm 11,1$ & 0,23 \\
\hline FM, kg/m & $15,0 \pm 8,3$ & $16,3 \pm 8,8$ & $13,7 \pm 8,1$ & 0,19 \\
\hline FFM, kg/m & $27,9 \pm 2,2$ & $28,1 \pm 2,1$ & $27,6 \pm 2,3$ & 0,83 \\
\hline TBW, \% & $50,5 \pm 7,5$ & $48,9 \pm 7,4$ & $52,0 \pm 7,6$ & 0,23 \\
\hline Waist Circ., cm & $88,6 \pm 13,2$ & $92,2 \pm 10,5$ & $85,3 \pm 15,0$ & 0,17 \\
\hline 6'Walking test, meters & $553,3 \pm 100,4$ & $556,0 \pm 120,6$ & $550,9 \pm 84,0$ & 0,91 \\
\hline Hip Circ., cm & $103,1 \pm 12,2$ & $104,2 \pm 9,0$ & $102,1 \pm 14,9$ & 0,71 \\
\hline Skin fold triceps, $\mathrm{cm}$ & $24,6 \pm 6,4$ & $24,9 \pm 7,1$ & $24,4 \pm 6,1$ & 0,88 \\
\hline Skin fold biceps, $\mathrm{cm}$ & $15,7 \pm 6,9$ & $17,8 \pm 7,5$ & $13,8 \pm 6,1$ & 0,19 \\
\hline Skin fold subscapular, cm & $20,8 \pm 6,1$ & $21,9 \pm 5,5$ & $19,9 \pm 6,7$ & 0,48 \\
\hline Skin fold supra-ilium, cm & $17,9 \pm 6,8$ & $18,6 \pm 6,2$ & $17,2 \pm 7,7$ & 0,67 \\
\hline Chair test, $n^{\circ}$ repetitions & $17,4 \pm 4,5$ & $18,2 \pm 5,1$ & $16,6 \pm 4,0$ & 0,44 \\
\hline Hand Grip test, kg & $\begin{array}{l}\text { DX } 22,7 \pm 3,9 \\
\text { SX } 22,9 \pm 4,2\end{array}$ & $\begin{array}{l}\text { DX 23,8 } \pm 3,9 \\
\text { SX } 23,2 \pm 3,4\end{array}$ & $\begin{array}{l}\text { DX } 21,8 \pm 3,8 \\
\text { SX } 22,6 \pm 5,0\end{array}$ & 0,33 \\
\hline MEDI LITE score & $13 \pm 2$ & $14 \pm 1$ & $12 \pm 3$ & 0,09 \\
\hline Weekly physical activity, minutes & $186 \pm 121$ & $174 \pm 81$ & $197 \pm 152$ & 0,78 \\
\hline
\end{tabular}

Data are reported as mean $\pm \mathrm{SD}$

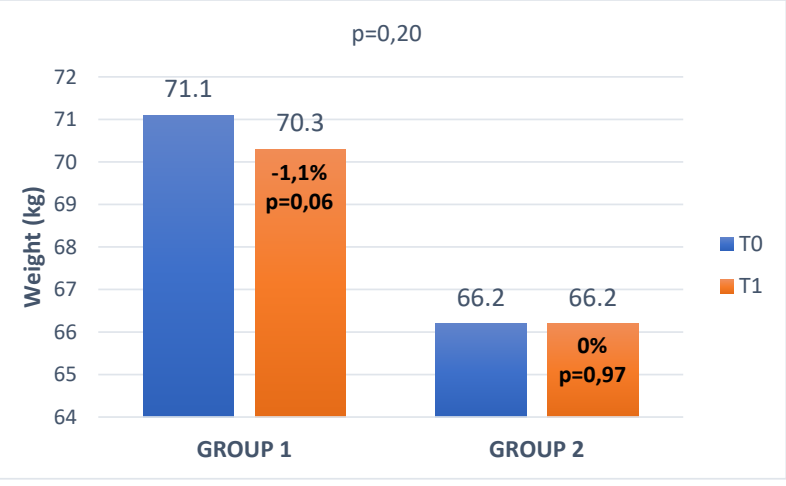

Figure 1. Comparison of weight behaviour in the groups studied after two months of intervention 
Table 2. Variations of the anthropometric, physical and eating habits parameters

\begin{tabular}{|c|c|c|c|c|c|c|}
\hline & G1 T0 & G1 T1 & $\mathbf{P}$ & G2 T0 & G2 T1 & $\mathbf{P}$ \\
\hline Weight, kg & $71,1 \pm 15,4$ & $70,3 \pm 14,8$ & 0,15 & $66,2 \pm 14,6$ & $66,2 \pm 14,0$ & 0,97 \\
\hline BMI, $\mathrm{kg} / \mathrm{m}^{2}$ & $28 \pm 5,9$ & $27,7 \pm 5,6$ & 0,11 & $25,8 \pm 6,1$ & $25,7 \pm 5,8$ & 0,36 \\
\hline FM, kg/m & $16,3 \pm 8,7$ & $16,3 \pm 8,6$ & 0,98 & $13,7 \pm 8,1$ & $14,2 \pm 7.2$ & 0,35 \\
\hline FFM, kg/m & $28,1 \pm 2,1$ & $28,0 \pm 2,1$ & 0,48 & $27,6 \pm 2,2$ & $26,9 \pm 2,2$ & 0,04 \\
\hline Waist Circ., cm & $92,2 \pm 10,5$ & $90,8 \pm 9,3$ & 0,05 & $85,3 \pm 15,0$ & $85,1 \pm 14,7$ & 0,72 \\
\hline Tricipital fold, $\mathbf{c m}$ & $24,9 \pm 7,2$ & $23,5 \pm 6,9$ & 0,04 & $24,5 \pm 6,1$ & $24,2 \pm 6,2$ & 0,34 \\
\hline Bicipital fold, $\mathrm{cm}$ & $15,6 \pm 7,8$ & $13,9 \pm 6,8$ & 0,02 & $13,9 \pm 6,1$ & $13,7 \pm 5,1$ & 0,73 \\
\hline Subscap. fold, cm & $21,8 \pm 5,6$ & $22,2 \pm 6,0$ & 0,29 & $19,0 \pm 5,6$ & $18,2 \pm 5,8$ & 0,04 \\
\hline Suprailiac fold, $\mathrm{cm}$ & $18,5 \pm 6,2$ & $17,7 \pm 6,1$ & 0,10 & $17,2 \pm 7,7$ & $17,5 \pm 7,7$ & 0,40 \\
\hline $\begin{array}{l}\text { Weekly physical } \\
\text { activity, minutes }\end{array}$ & $174 \pm 81$ & $196 \pm 100$ & 0,17 & $197 \pm 152$ & $193 \pm 148$ & 0,68 \\
\hline Chair test, $n^{\circ}$ & $18,2 \pm 5,1$ & $19,9 \pm 5,6$ & 0,02 & $16,6 \pm 4,0$ & $19,3 \pm 5,1$ & 0,01 \\
\hline Sit \& Reach test, cm & $4,9 \pm 5,7$ & $5,8 \pm 5,6$ & 0,24 & $0,8 \pm 7,3$ & $0,7 \pm 7,5$ & 0,93 \\
\hline 6MWT, m & $556 \pm 120$ & $553 \pm 97$ & 0,80 & $551 \pm 84$ & $545 \pm 91$ & 0,57 \\
\hline CR10 (0-10) & $4,6 \pm 1,9$ & $3,7 \pm 1,1$ & 0,07 & $4,2 \pm 2,5$ & $4,2 \pm 2,6$ & 0,98 \\
\hline Hand Grip dx, kg & $23,4 \pm 3,8$ & $24,3 \pm 3,8$ & 0,04 & $21,8 \pm 3,8$ & $22,2 \pm 4,0$ & 0,36 \\
\hline Hand Grip sx, kg & $23,2 \pm 3,4$ & $24,1 \pm 3,6$ & 0,20 & $22,6 \pm 5,0$ & $22,8 \pm 5,1$ & 0,67 \\
\hline MEDI LITE score & $13,5 \pm 1,3$ & $14,6 \pm 1,0$ & 0,02 & $12,0 \pm 2,7$ & $13,2 \pm 2,2$ & 0,01 \\
\hline
\end{tabular}

Data are reported as mean $\pm \mathrm{SD}$

previously by weight; $\mathrm{p}$ was equivalent to 0.11 and the $\mathrm{SD}=0.5 \mathrm{~kg} / \mathrm{m}^{2}$. In Group 2, the change was $0.1 \mathrm{~kg} / \mathrm{m}^{2}$, with $\mathrm{p}=0.36$ and $\mathrm{SD}=0.8 \mathrm{~kg} /$ $\mathrm{m}^{2}$. Therefore, for both groups, the modifications were not significant. Despite no significant changes were observed between the two groups $(p=0.97)$ however in the G1 the trend was evidently improved, while in the group 2 the trend was toward a worsening.

Group 1 showed significant improvements in the reduction of triceps and bicipital folds $(-1.4 \mathrm{~mm}, \mathrm{p}=0.04 ;-1.7 \mathrm{~mm}, \mathrm{p}=0.02)$; further decreases were found for the waist circumference $(-1.4 \mathrm{~cm}, \mathrm{p}$ $=0.05)$ and for the suprailiac fold $(-0.8 \mathrm{~mm}, \mathrm{p}=0.10)$. Instead, group 2 showed significant reductions in subscapular plica $(-0.8 \mathrm{~mm}, \mathrm{p}=$ $0.04)$ and fat free mass $(-0.8 \mathrm{~kg} / \mathrm{m}, \mathrm{p}=0.04)$; at the same time the fat mass increased, thus worsening the ratio between the two $(+0.5 \mathrm{~kg} / \mathrm{m}$, $\mathrm{p}=0.35)$. Significant difference between groups was observed for the subscapular fold $(\mathrm{p}=0.01)$.

About physical parameters, group 1 increased time dedicated to physical activity during the week by 21 minutes, with $\mathrm{p}=0.17$ and DS $=$ $37 \mathrm{~min}$, on the contrary Group 2 reduced physical activity by an average of 4 minutes, with $\mathrm{p}=0.68$ and DS $=44 \mathrm{~min}$. The difference between the two groups was not significant $(\mathrm{p}=0.12)$.

Group 1 showed a statistically significant increase in both the number of rises made $(+1.7, \mathrm{p}=0.02)$, assessed through the Chair Test, and the strength of the right hand $(+0.9 \mathrm{~kg} ; \mathrm{p}=0.04)$, calculated with the Hand Grip Test. For Group 2, changes in the parameters considered were less important. In fact, only a significant increase in the number of rises evaluated with the Chair Test was observed $(+2.6 ; \mathrm{p}=0.01)$. No statistically significant differences emerged between the two groups.

Finally, two months after the delivery of the ten WCRF/ AICR recommendations for Group 1 and the standardized dietary recommendations for Group 2, a significant increase in adherence to the Mediterranean diet was observed in both intervention groups. In fact, Group 1 increased its adherence by 1.1 points, with $\mathrm{p}=0.02$ and DS $=1.0$, thus reaching 14.6 points out of a maximum of 18; Group 2 improved adherence by 1.2 points, with $\mathrm{p}=0.01$ and $\mathrm{DS}=1.2$, reaching 13.2 points out of 18 . No significant differences emerged between the two groups $(\mathrm{p}=0.94)$.

\section{Discussion}

Cancer prevention is one of the most relevant aspect in the continuous improvement of the progression of the metabolic chronic disease in our society and in the world. The WCRF recommendations support the evidence of a structured approach to reduce the fragmentation in the life style reconditioning in cancer protocol [8].

After two months of follow up, in this population of Italian survived breast cancer patients, participants of the group 1 had a global improvement for both anthropometric and physical parameters compared with that of participants of the group 2, who did not change their lifestyle due to lack of administration of WCRF recommendations.

For group 1, in fact, the increase in the amount of weekly physical activity combined with the significant improvement in adherence to the Mediterranean diet, has allowed to reduce both the weight and the BMI of the participants. This did not happen for group 2 .

In fact, obesity is related to the increased risk of 11 types of cancer, including breast cancer [9]. The achievement of the normal weight combined with the maintenance of muscle mass are two essential requirements to reduce the risk of contracting a new tumoral pathology and at the same time maintaining a good quality of life; for this reason the analysis of body composition through bioimpedentiometry has proved to be fundamental.

Diet, nutrition and physical activity recommendations are an integrated process by which the cells tissues and all the organism take energy. Many hypotheses support the role of tumour metabolism as well as the tumour-host interaction that may be selectively influenced by single bouts as well as regularly applied exercise, dependent on exercise intensity, duration, frequency and mode [10].

Lactate metabolism has been shown to be directly correlated with the prognosis of cancer: in fact, accelerated glucose consumption is associated with tumorigenesis [11]. Anthropometric measurements have been in fact largely considered for their peculiar indications to correctly monitor the patient with an increased cardiovascular risk as in case of cancer. 


\section{Conclusions}

The study aims to underline the importance of constant adherence to the WCRF/AICR for a correct management of eating habits. The pilot study is aimed at overcoming the general suggestions of the Mediterranean dietary indications. This approach seems to be crucial for achieving a significant impact on the main cardiovascular risk parameters. Despite the data obtained are in agreement with the literature and support this hypothesis, however the studied sample is limited, and the time of the exploration is limited to a short period. Furthermore, no specific investigation of the potential impact of the different intensity of individual physical activity was considered as an independent variable in determining the possible differences in the investigated groups. In the future, more attention will be needed to evaluate the correct and adequate follow-up of the intensity of daily physical activity.

In this term, it can be helpful to investigate the possible background of a constant physical activity in the past, which can contribute to a more constant adhesion during this type of approach. It is sensible to promote these recommendations in cancer, especially when regular and individual exercise is allowed and constantly practiced.

The results obtained in this pilot study confirm the applicability of this approach in a cancer population with an effective significant improvement with respect of the subjects underwent to the general indications. In addition to all this, bioimpedance assessment (BIA), based on the principle that resistance to an electric current is greater in adipose tissue than in lean tissue, has allowed defining in more detail the body composition of each patient.

It seems that especially the breast cancer patients are motivated by the knowledge that the breast cancer is now considered a chronic disease with a long expectance of life and therefore with a potential major positive impact from this approach. A structured program including WCRF/AICR the needs to be shared for cancer survived population.

\section{Acknowledgements}

The authors certify that there is no conflict of interest with any financial organization regarding the material discussed in the manuscript.

Authors' contributions: SM has conceived the study, collected the data, has contributed to the statistical analysis and to the drafting of the paper; LS has written the paper and planned the study; FS has planned the study, analysed the data and edit the paper for the implementation of the message; GG has participated and approved to improve the message of the study.

\section{References}

1. Lozano R, Naghavi M, Foreman K, Lim S, Shibuya K, et al. (2012) Global and regional mortality from 235 causes of death for 20 age groups in 1990 and 2010: a systematic analysis for the Global Burden of Disease Study 2010. Lancet 380: 2095-128. [Crossref]

2. Panico S, Mattiello A, Panico C, Chiodini P (2014) Mediterranean Dietary Pattern and Chronic Diseases. Cancer Treat Res 159: 69-81. [Crossref]

3. Playdon MC, Bracken MB, Sanft TB, Ligibel JA, Harrigan M, et al. (2015) Weight Gain After Breast Cancer Diagnosis and All-Cause Mortality: Systematic Review and Meta-Analysis. J Natl Cancer Inst 107: djv275. [Crossref]

4. Sofi F, Dinu M, Pagliai G, Marcucci R, Casini A (2017) Validation of a literature-based adherence score to Mediterranean diet: the MEDI-LITE score. Int J Food Sci Nutr 68 757-762. [Crossref]

5. Sofi F, Macchi C, Abbate R, Gensini GF, Casini A (2014) Mediterranean diet and health status: an updated meta-analysis and a proposal for a literature-based adherence score. Public Health Nutr 17: 2769-2782. [Crossref]

6. Aristizábal JC, Restrepo MT, Estrada A (2007) Body composition assessment by anthropometry and bioelectrical impedance. Biomedica 27: 216-224. [Crossref]

7. Brzycki M (1998) A practical approach to strength training. Chicago: McGraw-Hill

8. Kohler LN, Garcia DO, Harris RB, Oren E, Roe DJ, et al. (2016) Adherence to Diet and Physical Activity Cancer Prevention Guidelines and Cancer Outcomes: A Systematic Review. Cancer Epidemiol Biomarkers Prev 25: 1018-1028. [Crossref]

9. Ackerman SE, Blackburn OA, Marchildon F, Cohen P (2017) Insights into the Link Between Obesity and Cancer. Curr Obes Rep 6: 195-203. [Crossref]

10. Brown JC, Winters-Stone K, Lee A, Schmitz KH (2012) Cancer, physical activity, and exercise. Compr Physiol 2: 2775-2809. [Crossref]

11. Wu H, Ying M, Hu X (2016) Lactic acidosis switches cancer cells from aerobic glycolysis back to dominant oxidative phosphorylation. Oncotarget 7: 40621-40629. [Crossref]

Copyright: (C2019 Magro S. This is an open-access article distributed under the terms of the Creative Commons Attribution License, which permits unrestricted use, distribution, and reproduction in any medium, provided the original author and source are credited. 